\title{
Impacto da dor, estado antropométrico e fatores associados em pacientes com enxaqueca
}

\author{
Impact of pain, anthropometric status and associated factors in patients with migraine
}

\section{DOI: $10.37111 /$ braspenj.2019344015}

Luana de Oliveira Leite ${ }^{1}$

Lorraine Lacerda Brasil Souza ${ }^{2}$

Júlia Canto e Sousa ${ }^{3}$

Sara Factum Dutra ${ }^{4}$

\section{Unitermos: \\ Transtornos de enxaqueca. Cefaleia. Estado nutricio- nal. Antropometria.}

\section{Keywords:}

Migraine disorders. Headache. Nutritional status. Anthropometry.

\section{Endereço para correspondência:} Luana de Oliveira Leite

Departamento de Ciências da Vida, Universidade do Estado da Bahia (UNEB)

Rua Silveira Martins, 2555 - Cabula - Salvador, BA Brasil - CEP 41150-000

E-mail: luanaleite_nutri@yahoo.com.br

\section{Submissão}

12 de julho de 2019

Aceito para publicação

13 de novembro de 2019

\begin{abstract}
RESUMO
Introdução: A enxaqueca, ou migrânea, é uma doença neurológica crônica, que apresenta diversos fatores etiológicos. Destaca-se a importância de estudos que envolvam os pacientes com migrânea, já que esta gera significativas limitações na qualidade de vida. Objetivos: Avaliar a associação entre impacto da dor, estado antropométrico e outros fatores em pacientes com enxaqueca. Método: Estudo do tipo quantitativo e descritivo, de corte transversal e de base secundária, desenvolvido numa Clínica Escola de Farmácia da cidade de Salvador-BA, com pacientes atendidos com diagnóstico de enxaqueca, no período de abril de 2018 a março de 2019, com idade igual ou superior a 20 anos. Foram coletadas informações demográficas, socioeconômicas, antropométricas, estilo de vida, clínicas, impacto da dor [pelos questionários Migraine Disability Assessment (MIDAS) e Headache Impact Test-6 (HIT-6)], e intensidade da dor (escala visual da dor). Modelos de regressão logística foram empregados para avaliar os fatores associados ao impacto da dor, com valor de $p \leq 0,05$. Resultados: Dos 40 pacientes avaliados, $87,5 \%$ eram do sexo feminino, a média de idade detectada foi 40,35 $\pm 12,6$ anos. Observou-se prevalência de sedentários $(87,5 \%)$, excesso de peso $(55 \%)$, circunferência da cintura elevada $(68,4 \%)$, obstipação $(35 \%)$ e intolerância à lactose $(28,2 \%)$, alto impacto nas atividades cotidianas: $62,5 \%$ (HIT-6) e $69,6 \%$ (MIDAS). Maiores médias de índice de massa corporal (IMC) e circunferência da cintura (CC) foram identificadas nos pacientes com substancial/muito importante impacto da dor $(p=0,03$ e $p=0,06$, respectivamente) em relação aos com nenhum/algum impacto. As chances de maior impacto da dor por meio do HIT-6 parecem estar associadas ao excesso de peso $(p=0,04)$, sexo feminino $(p=0,62)$ e sedentarismo $(p=0,89)$. Conclusões: Estado antropométrico associou-se ao impacto da enxaqueca. Excesso de peso e o sexo feminino estão relacionados à ocorrência de enxaqueca e, conjuntamente com o sedentarismo, parecem influenciar no impacto da dor.
\end{abstract}

\section{ABSTRACT}

Introduction: Migraine is a chronic neurological disease which has various etiological factors. It is important to highlight the importance of studies involving patients with migraine, as it generates significant limitations in quality of life. Objectives: To evaluate the association among pain impact, anthropometric status and others factors in migraine patients. Methods: Quantitative and descriptive, cross-sectional and secondary-based study, developed in a Clinical School of Pharmacy of the city of Salvador-BA, with patients treated with migraine diagnosis, from April 2018 to March 2019 , of 20 years old or more. Information was gathered about demographic, socioeconomic, anthropometric, lifestyle, clinical, pain impact [by Migraine Disability Assessment (MIDAS) and Headache Impact Test-6 questionnaires (HIT-6)] and pain intensity (visual pain scale). Logistic regression models were used to evaluate the factors associated with the pain impact with a value of $p \leq 0.05$. Results: Of the 40 patients evaluated, $87.5 \%$ were female, the average age detected was $40.35 \pm 12.6$ years. There was a prevalence of sedentary $(87.5 \%)$, overweight $(55 \%)$, high waist circumference $(68.4 \%)$, intestinal constipation (35\%) and lactose intolerance $(28.2 \%)$; high impact on daily activities: $62.5 \%$ (HIT-6) and $69.6 \%$ (MIDAS). Higher body mass index (BMI) and waist circumference (WC) averages were identified in patients with substantial/severe pain impact ( $p=0.03$ and $p=0.06$, respectively) compared to those with no/some impact. The chances of greater impact of pain through HIT-6 seem to be associated with overweight $(p=0.04)$, female sex $(p=0.62)$ and physical inactivity $(p=0.89)$. Conclusion: Anthropometric status was associated with the impact of migraine. Overweight and female sex are related to migraine occurrence, and together with physical inactivity seem to influence the pain impact.

1. Nutricionista; Doutoranda e Mestre no Programa de Pós-graduação em Alimentos, Nutrição e Saúde da Universidade Federal da Bahia (UFBA); Docente do Curso de Bacharelado em Nutrição, Departamento de Ciências da Vida, Universidade do Estado da Bahia (UNEB), Salvador, BA, Brasil.

2. Nutricionista; Graduada no curso de Bacharelado em Nutrição da Universidade do Estado da Bahia, Salvador, BA, Brasil.

3. Nutricionista; Pós-graduanda em Nutrição Clínica: Metabolismo, Prática e Terapia Nutricional na Universidade Estácio de Sá, Rio de Janeiro, RJ, Brasil.

4. Nutricionista; Pós-graduanda em Nutrição de Pacientes com Enfermidades Renais no Instituto Cristina Martins de Educação em Saúde, Curitiba, PR, Brasil. 


\section{INTRODUÇÃO}

A enxaqueca, ou migrânea, é uma desordem neurológica crônica marcada especialmente por cefaleia, mas também por hipersensibilidade a estímulos visuais, auditivos, olfativos e cutâneos, náusea e vômito' . De acordo com a $3^{a}$ edição do International Classification of Headache Disorders (ICHD), a enxaqueca crônica é diagnosticada quando o paciente apresenta cefaleia por 15 dias ou mais no mês, ocorrendo também episódios dos demais aspectos característicos da enxaqueca em pelo menos 8 deles ${ }^{2}$. $O$ tratamento inclui a modificação de fatores de risco, bem como evitar os fatores desencadeantes, além do tratamento farmacológico e não-farmacológico que visa prevenir e cessar as crises da enxaqueca'.

Com prevalência mundial de aproximadamente 11,5\% e, no Brasil, de $15 \%{ }^{3}$, a migrânea é classificada como uma das principais condições incapacitantes em todo o mundo, especialmente em indivíduos com idade abaixo de 50 $a^{a n o s}{ }^{4}$. Torna-se evidente, portanto, o impacto da enxaqueca da qualidade de vida dos pacientes, especialmente ao considerar-se que a faixa etária de maior incidência é mais frequentemente relacionada com a realização de atividades laborais e familiares ${ }^{5}$.

Sendo caracterizada como uma doença de etiologia diversa, diferentes aspectos se relacionam com a fisiopatologia da migrânea. Dentre eles, o estilo de vida é evidenciado nas estratégias de manejo desta condição $0^{6}$. A prática de atividade física demonstra ligação, porém ainda controversa e pouco esclarecida ${ }^{7}$. Assim como a idade, o sexo também é identificado como um fator que afeta a associação entre a enxaqueca e peso corporal, mostrando-se mais prevalente no sexo feminino ${ }^{8}$. $O$ aumento do peso corporal é apontado como um importante fator de risco em diversos trabalhos ${ }^{8-10}$.

Tendo em vista o impacto importante da migrânea na qualidade de vida dos pacientes acometidos e a estreita relação desta condição com o estado antropométrico, sexo e estilo de vida, destaca-se a importância de estudos que envolvam essa população, com o intuito de fornecer informações que poderão ser utilizadas não só na prevenção, mas também como auxílio na tomada de decisões na conduta nutricional.

Diante do exposto, o presente trabalho tem como objetivo avaliar a associação entre impacto da dor, estado antropométrico, fatores demográficos e de estilo de vida em pacientes com enxaqueca.

\section{MÉTODO}

Estudo do tipo quantitativo, com abordagem analíticodescritiva, de corte transversal, retrospectivo e de base secundária desenvolvido numa clínica escola de Farmácia, como parte de um projeto interdisciplinar "Projeto Interdisciplinar de Atenção à Saúde ao Portador de Enxaqueca", de caráter permanente pertencente ao Departamento de Ciências da Vida (DCV), da Universidade do Estado da Bahia (UNEB), Campus I, Salvador, Bahia.

Obtiveram-se dados dos prontuários de pacientes atendidos durante o período de abril de 2018 a março de 2019, com coleta de dados em março de 2019. Incluiu-se pacientes com idade igual ou acima de 20 anos e com diagnóstico clínico de enxaqueca. Pacientes com idade inferior a 20 anos, gestantes e nutrizes não foram incluídos no estudo. Esta pesquisa foi aprovada por Comitê de Ética em Pesquisa envolvendo seres humanos da UNEB, sob parecer número 3.255.056, em 10 de abril de 2019.

As informações coletadas dos prontuários foram obtidas durante a consulta nutricional por nutricionistas e estagiários treinados. Coletou-se informações demográficas - sexo e idade (20 a 59 anos e $\geq 60$ anos); socioeconômicas escolaridade (analfabeto, ensino fundamental completo ou incompleto, ensino médio completo ou incompleto e ensino superior completo ou incompleto) e renda familiar mensal (< 1 salário mínimo, entre 1 e 2 salários mínimos ou $\geq 3$ salários mínimos); além de estilo de vida (atividade física).

As informações antropométricas foram obtidas de acordo com técnicas preconizadas na literatura ${ }^{11}$. Coletou-se dados sobre peso em quilogramas - medido com balança tipo plataforma eletrônica da marca OMRON, com capacidade de $150 \mathrm{~kg}$ e precisão de $100 \mathrm{~g}$; altura em metros - obtida com estadiômetro vertical da marca Welmy ${ }^{\circledR}$ com precisão de 0,1 cm; circunferência da cintura (CC) - utilizando fita métrica inelástica com precisão de $0,1 \mathrm{~cm}$.

O índice de massa corporal (IMC=peso/altura $\left.{ }^{2}\right)$ foi calculado a partir dos dados de peso e altura. Seguiu-se os critérios da Organização Mundial da Saúde (OMS) ${ }^{12}$, para classificação dos adultos, e os critérios da Nutrition Screening Initiative - NSI ${ }^{13}$, para classificação dos idosos (igual ou maior que 60 anos). Para classificar a obesidade abdominal a partir da CC em adultos e idosos, utilizou-se os pontos de corte sugeridos pela $\mathrm{OMS}^{14}$. Esses dados descritos determinaram o estado antropométrico da amostra neste estudo.

Foram coletadas informações clínicas referentes ao diagnóstico de intolerância à lactose (realizado por meio de teste sanguíneo). Quanto ao diagnóstico de enxaqueca, foi realizado por neurologista da clínica durante a triagem para o projeto, tendo como parâmetro as diretrizes da International Headache Society ${ }^{2}$. Coletou-se, ainda, o tempo de diagnóstico da doença (em anos), o nível da intensidade da dor e o grau do impacto da dor.

Utilizou-se a escala visual analógica (9) validada para avaliar a intensidade da dor. A partir desta, o paciente analisa a intensidade de seus sintomas em uma escala de 0 a 10, subdividida em 0-2 = dor leve; 3-7 = dor moderada; $8-10=$ dor intensa $^{15}$. 
Para avaliar o impacto da dor, utilizou-se dois questionários validados:

1 - Migraine Disability Assessment (MIDAS): Quantifica em número de dias perdidos durante um período de 90 dias, a incapacidade gerada pela cefaleia nas atividades sociais, produtivas e trabalhistas. A somatória dos dias é representada por um escore subdividido em graus; o grau I (0 a 5$)$ = nenhuma ou pouca incapacidade; o grau II $(6$ a 10) = leve incapacidade; o grau III (1 1 a 20) = moderada incapacidade; e o grau IV $(\geq 21)$ = intensa incapacidade ${ }^{16}$.

2- Headache Impact Test-6 (HIT-6): Composto por seis questões que avaliam a gravidade da dor, perdas de dias de trabalho e atividades sociais, além de alterações cognitivas e de humor. A somatória dos pontos é subdividida em intervalos: $<50$ pontos $=$ pouco ou nenhum impacto; 50 a 55 pontos = algum impacto; 56 a 59 pontos $=$ impacto substancial $; \geq 60$ pontos $=$ impacto muito importante ${ }^{17}$.

Para o diagnóstico de obstipação, seguiu-se os Critérios de Roma III18, o qual considera obstipados todos os indivíduos que, no período dos últimos três meses, apresentaram os seguintes indicadores (2 ou mais): 1 . Esforço para evacuar em pelo menos $25 \%$ das vezes; 2 . Fezes duras ou irregulares em pelo menos $25 \%$ das defecações; 3 . Sensação de evacuação incompleta em pelo menos $25 \%$ das vezes; 4 . Sensação de obstrução anorretal em pelo menos $25 \%$ das defecações; 5 . Manobras manuais para facilitar a defecação em pelo menos $25 \%$ das vezes; 6 . $\leq 3$ defecações por semana.

Todos os dados foram organizados em planilha do programa da Microsoft Office Excel versão 2013, na qual também se realizou os cálculos do IMC.

Os resultados das variáveis categóricas foram apresentados em frequências absolutas (n) e frequências relativas (\%) e das contínuas em valores médios e desvio padrão. Foi utilizado o teste $t$ de Student para detectar diferença de médias entre amostras independentes, considerando-se a distribuição normal dos dados, com valor de $p \leq 0,05$, indicando significância estatística. Modelos de regressão logística foram realizados para avaliar os fatores (sexo, idade, IMC e atividade física) associados ao impacto da dor nos pacientes avaliados, que foram expressos em odds ratios (OR) e seus respectivos intervalos de confiança (IC) de 95\%, considerando significância estatística quando $p \leq 0,05$. Foi utilizado o software estatístico SPSS Statistic versão 20.0.0 para análise dos dados.

\section{RESULTADOS}

Na Tabela 1, estão apresentadas as características demográficas, socioeconômicas, de estilo de vida, antropométricas e clínicas da população estudada. A amostra foi composta por 40 prontuários. Nesse estudo, predominouse o sexo feminino $(87,5 \%)$; a idade variou entre 20 e 65
Tabela 1 - Caracterização segundo variáveis demográficas, socioeconômicas, de estilo de vida, antropométricas e clínicas de pacientes com enxaqueca atendidos numa clínica escola de Farmácia. Salvador-BA, 2019.

\begin{tabular}{lcc}
\hline Variável & N & $\%$ \\
\hline Sexo & 355 & 87,5 \\
Feminino & & 12,5 \\
Masculino & & \\
\hline Idade & 38 & 95,0 \\
Adulto ( $\geq 20$ e <60 anos) & 2 & 5,0 \\
Idoso ( 60 anos) & & \\
\hline Escolaridade & - & - \\
Analfabeto & 3 & 7,9 \\
Ensino fundamental & 181 & 47,4 \\
Ensino médio & 7 & 44,7 \\
Ensino superior & & \\
\hline Renda familiar & 8 & 22,2 \\
$<1$ salário mínimo & 18 & 50,0 \\
1 - 2 salários mínimos & 10 & 27,8 \\
\hline 3 salários mínimos
\end{tabular}

\begin{tabular}{lcc}
\hline Atividade física & & \\
Sim & 5 & 12,5 \\
Não & 35 & 87,5 \\
\hline IMC & & \\
Abaixo do peso & 1 & 2,5 \\
Peso adequado & 172 & 42,5 \\
Excesso de peso & 2 & 55,0 \\
\hline CC & & \\
Adequada & 122 & 31,6 \\
Inadequada & 6 & 68,4 \\
\hline Obstipação & & \\
Sim & 142 & 35,0 \\
Não & 6 & 65,0 \\
\hline In
\end{tabular}

\begin{tabular}{lcc}
\hline Intolerância à lactose & & \\
Sim & 112 & 71,8 \\
Não & 8 & 28,2 \\
\hline HIT-6 & 7 & \\
Pouco ou nenhum impacto & 3 & 9,9 \\
Algum impacto & 2 & 9,4 \\
Impacto substancial & 20 & 62,5 \\
Impacto muito importante & & \\
\hline MIDAS & 2 & 8,7 \\
Nenhuma ou pouca incapacidade & 1 & 4,3 \\
Leve incapacidade & 4 & 17,4 \\
Moderada incapacidade & 16 & 69,6 \\
Intensa incapacidade & & \\
EVA & - & - \\
Dor leve & 9 & 27,3 \\
Dor moderada & 24 & 72,7 \\
Dor intensa &
\end{tabular}

IMC = Índice de massa corporal; CC = Circunferência da cintura; HIT-6 = Headache Impact Test-6; MIDAS = Migraine Disability Assessment; EVA = Escala visual analógica. 
anos, com média de 40,35 $\pm 12,6$ anos e predominância da faixa etária adulta $(95,0 \%)$; todos os participantes eram escolarizados, sendo a maioria $(47,4 \%)$ com ensino médio; a renda familiar foi prevalente entre 1 e 2 salários mínimos compondo $50 \%$ da população; os sedentários constituíram $87,5 \%$ da amostra. Quanto ao estado antropométrico, o excesso de peso, segundo o IMC, e a obesidade abdominal, segundo a CC, foram de $55 \%$ e $68,4 \%$, respectivamente. Obstipação e intolerância à lactose foram encontradas em $35 \%$ e $28,2 \%$ dos pacientes assistidos, respectivamente.

Em relação aos parâmetros analisados sobre a enxaqueca, a maioria da amostra apresentou impacto muito importante de dor (62,5\%); intensa incapacidade de dor $(69,6 \%)$ e dor intensa $(72,7 \%)$, segundo os questionários HIT-6, MIDAS e a EVA, respectivamente.

$\mathrm{Na}$ Tabela 2, identificou-se maior média de IMC nos pacientes cujo HIT-6 indicou impacto substancial/importante da enxaqueca $\left(29,7 \pm 6,2 \mathrm{~kg} / \mathrm{m}^{2}\right)$ em relação àqueles que o HIT-6 identificou nenhum/algum impacto $(25,1 \pm 4,7 \mathrm{~kg} /$ $\left.\mathrm{m}^{2}\right)$, sendo que essa diferença de médias foi estatisticamente significante $(p=0,03)$. De forma similar, maior média de CC foi observada nos indivíduos também com pontuação do HIT-6, identificando impacto substancial/importante $(95,9 \pm$ $14,4 \mathrm{~cm})$ em contraponto aos que o impacto foi ausente ou baixo $(85,3 \pm 13,7 \mathrm{~cm})$, detectando-se tendência para essa associação $(p=0,06)$.

Modelos de regressão logística, avaliando fatores associados ao impacto da dor, pelo questionário HIT-6, nos pacientes estudados estão representados na Tabela 3. Observou-se que, nos pacientes avaliados nesse estudo, o excesso de peso aumentou em 19\% a chance de maior impacto da dor com valor de $p$ indicando significância estatística $(p=0,04)$; o sexo masculino e a prática de atividade física diminuíram em $50 \%$ e $17 \%$, respectivamente, a chance de maior impacto da enxaqueca em comparação aos indivíduos do sexo feminino e sedentários, embora esses resultados não tenham sido estatisticamente significantes.

Tabela 2 - Associação entre impacto da dor (HIT-6) e estado antropométrico em pacientes atendidos numa clínica escola de Farmácia. Salvador-BA, 2019.

\begin{tabular}{|c|c|c|c|c|}
\hline \multirow[b]{2}{*}{$\begin{array}{l}\text { Variáveis } \\
\text { antropo- } \\
\text { métricas } \\
\text { relacionadas à } \\
\text { enxaqueca }\end{array}$} & \multirow[b]{2}{*}{$\begin{array}{c}\text { Total } \\
\text { Média } \\
\text { ( } \pm \text { desvio padrão) }\end{array}$} & \multicolumn{2}{|c|}{$\begin{array}{c}\text { HIT - } 6 \text { (pontos) } \\
\text { Média ( } \pm \text { desvio padrão) }\end{array}$} & \multirow[b]{2}{*}{$\begin{array}{c}\text { Valor } \\
\text { de } \\
p^{*}\end{array}$} \\
\hline & & $\begin{array}{l}\text { Substancial/ } \\
\text { importante } \\
\text { impacto }\end{array}$ & $\begin{array}{c}\text { Nenhum/ } \\
\text { algum } \\
\text { impacto }\end{array}$ & \\
\hline IMC & $26,78 \pm 5,24$ & $29,66 \pm 6,24$ & $25,11 \pm 4,75$ & 0,03 \\
\hline $\mathrm{CC}$ & $88,28 \pm 13,75$ & $95,95 \pm 14,40$ & $85,33 \pm 13,74$ & 0,06 \\
\hline
\end{tabular}

Valores em média \pm desvio padrão; HIT-6 = Headache Impact Test-6; IMC = índice de massa corporal; $\mathrm{CC}=$ circunferência da cintura.

*valor de p compara valores de médias entre grupos com substancial/severo e com nenhum/ algum impacto da enxaqueca, usando Test T para amostras independentes
Tabela 3 - Modelos de regressão logística avaliando fatores associados ao impacto da enxaqueca pelo questionário HIT-6 em pacientes atendidos numa clínica escola de Farmácia. Salvador-BA, 2019.

\begin{tabular}{lccc}
\hline Modelo & $\begin{array}{c}\text { Fatores Associados } \\
\text { (categorização) }\end{array}$ & OR (IC 95\%) & P-valor \\
\hline 1 & IMC (1= excesso de peso; & $1,19(1,00-1,40)$ & 0,04 \\
2 = sem excesso de peso) & & \\
& $\begin{array}{c}\text { Sexo (1= masculino; } \\
2=\text { feminino) }\end{array}$ & $0,50(0,32-7,70)$ & 0,62 \\
3 & $\begin{array}{c}\text { Atividade física } \\
\text { (1= sedentário; } 2=\text { ativo) }\end{array}$ & $0,83(0,56-12,38)$ & 0,89 \\
& & \\
\hline VIMC = Índice de massa corporal; OR = Odds ratio; IC = Intervalo de confiança.
\end{tabular}

\section{DISCUSSÃO}

Este estudo avaliou a associação entre impacto da dor pelo questionário HIT-6, estado antropométrico, sexo e prática de atividade física em pacientes com enxaqueca, identificando associação nos pacientes avaliados entre estado antropométrico e impacto substancial/importante da dor, além de importantes achados em relação ao excesso de peso, sexo feminino e sedentarismo como fatores de risco para impacto da enxaqueca.

O sexo feminino foi predominante na amostra estudada, refletindo a maior prevalência da enxaqueca em mulheres, sendo esta de 3 a 4 vezes mais comum neste sexo do que em homens ${ }^{3,19}$. Com as análises do presente estudo, foi demonstrado também que o sexo feminino é ainda um fator de risco para o maior impacto desta condição clínica, aumentando as chances de o paciente ter impacto substancial/importante da enxaqueca, quando avaliado pelo HIT-6.

A prevalência do sexo feminino está intimamente relacionada a fatores hormonais, uma vez que cerca de $50 \%$ das mulheres referem crises de enxaqueca associada ao período menstrual, seja antes, durante ou logo após seu término ${ }^{20}$. A prevalência da enxaqueca é semelhante em jovens de ambos os sexos, até a idade da puberdade, quando passa a se tornar mais comum em mulheres ${ }^{19}$. $O$ gatilho para a enxaqueca menstrual são os baixos níveis de estrogênio circulante, durante 2 a 3 dias antes do início da menstruação, na fase pré-menstrual ${ }^{20}$.

Dentre os resultados encontrados no estudo, outra variável analisada foi a faixa etária, evidenciando a predominância de adultos (20 a 65 anos) entre os indivíduos avaliados, achado correspondente ao encontrado em outros estudos que abordam a incidência e prevalência da enxaqueca ${ }^{19,21}$. Pelo seu potencial impacto, o caráter incapacitante desta condição clínica ocasiona ainda significativas limitações na qualidade de vida destes pacientes em relação à população saudável ${ }^{19}$.

O comprometimento no desempenho de atividades diárias, além da maior taxa de absenteísmo, em comparação a outras condições, evidencia seu impacto econômico. 
Segundo análise sistemática do Global Burden of Disease $2016^{4}$, enxaquecas são particularmente mais onerosas para pessoas de 15 a 49 anos, sendo uma das causas para anos vividos de forma incapacitante por esta população. Tal comprometimento foi refletido no presente estudo, onde observou-se grande prevalência de pontuações elevadas nos questionários de migrânea, com maioria da amostra apresentando impacto muito importante de dor, segundo HIT-6 e intensa incapacidade de dor, segundo MIDAS, assim como alta prevalência de dor intensa apontada na EVA. É possível notar, portanto, a característica debilitante da migrânea e a sua influência negativa na qualidade de vida dos pacientes.

Identificou-se predominância de sedentarismo, em detrimento aos praticantes de atividade física, evidenciando a importância deste fator de risco. Além disso, ainda foi identificado que o sedentarismo aumenta as chances de o paciente ter impacto substancial/importante da enxaqueca analisado pelo HIT-6, evidenciando a inatividade física como um dos agravantes para essa condição. Em um estudo intervencionista sueco, com indivíduos sedentários com enxaqueca, foi demonstrado que a prática de atividade aeróbica regular levou a redução significativa na frequência e intensidade das dores de cabeça crônicas e das crises de enxaqueca. $\bigcirc$ programa de treinamento aplicado consistia em treinos de 40 minutos de bicicleta ergométrica, três vezes por semana. Ao final das 12 semanas, além da redução das crises, a necessidade do uso de analgésicos também diminuiu ${ }^{22}$.

Não apenas o exercício aeróbico contribui para a redução de frequência e intensidade dos episódios de dor. Um estudo dinamarquês, que investigou a existência de associações específicas entre enxaqueca, sexo, estilo de vida e fatores socioeconômicos, demonstrou que há diminuição significativa do risco de enxaqueca associada à prática de exercício físico isométrico ${ }^{23}$. A analgesia induzida por exercício físico regular e não extenuante envolve a ativação de vias inibitórias centrais, ao reduzir a expressão dos transportadores de serotonina, aumentando seus níveis. O efeito opioide da serotonina atua na substância cinzenta periqueadutal e no núcleo ventromedial da medula espinhal, utilizando vias endógenas dos sistemas inibitórios para efeito analgésico, reduzindo assim a dor provocada pela enxaqueca ${ }^{24}$. A amostra deste estudo foi analisada, ainda, quanto à presença de obstipação e intolerância à lactose nestes pacientes, revelando que estas desordens gastrointestinais parecem ser mais frequentes em pacientes com enxaqueca em comparação à população em geral. Náuseas, vômitos, obstipação e dores abdominais são alguns dos sintomas gastrointestinais mais comuns durante as crises $^{25}$. O desequilíbrio da microbiota intestinal, aspecto patológico comum entre enxaqueca, obstipação e intolerância à lactose, parece ser o gatilho para essas condições clínicas. Em relação à enxaqueca, a disbiose, por alterar a permeabilidade intestinal, consequentemente, também aumenta a atividade inflamatória, contribuindo assim para a ativação do sistema trigeminovascular, o que resultaria na vasodilatação intracraniana, característica da migrânea ${ }^{26,27}$.

Um importante achado foi a associação entre maior média de IMC em pacientes com impacto substancial/ importante da enxaqueca, avaliado pelo HIT-6, bem como uma tendência para a associação com a CC. Soma-se a isso que o excesso de peso foi detectado como fator de risco, aumentando a chance de forma significativa de impacto substancial/importante da migrânea. De forma similar, um estudo conduzido a partir dos dados do ELSA-Brasil investigou a relação entre migrânea, obesidade - classificada a partir do IMC, e obesidade abdominal - mensurada por CC, encontrando uma forte associação entre maiores valores de IMC e migrânea diária, mas não com a obesidade abdominal ${ }^{10}$.

Peterlin et al. 9 , em um estudo utilizando dados do National Health and Nutrition Examination Survey (NHANES), concluíram que a prevalência de migrânea em homens e mulheres com idade $\leq 55$ anos foi maior naqueles com elevada gordura corporal, o mesmo foi observado em indivíduos com obesidade abdominal. Um estudo transversal iraniano também observou que a obesidade central foi positivamente associada com a frequência e intensidade das crises de enxaqueca ${ }^{28}$.

Existem evidências que demonstram a associação entre a migrânea e o hipotálamo, onde encontra-se o centro de controle da fome, bem como de seus peptídeos, proteínas e neurotransmissores associados à fome, podendo levar à hiperfagia e ganho de peso. Outro mecanismo proposto é o aumento da expressão de citocinas pró-inflamatórias, como as interleucinas 1 e 6 (IL- 1 e IL-6) e o fator de necrose tumoral (TNF) - $\alpha$, em decorrência da expansão do tecido adiposo durante o ganho de peso. Alterações nos níveis dessas citocinas também já foram observados em paciente com migrânea ${ }^{29}$. É notável a influência da obesidade na frequência e intensidade da migrânea. Ambas são condições multifatoriais, cujos mecanismos envolvidos podem ser afetados pela perda de peso corporal ${ }^{30}$. No entanto, são necessários mais estudos que elucidem com maior precisão a interrelação entre obesidade e enxaqueca.

Por se tratar de um estudo transversal, há limitações quanto à identificação das relações de causa e efeito, além do reduzido tamanho amostral, impossibilitando, portanto, a realização de análises inferenciais de associação estatística. Contudo, essas limitações não comprometem a qualidade deste trabalho e nem as observações encontradas.

\section{CONCLUSÃO}

No presente estudo, identificamos o predomínio de enxaqueca entre indivíduos do sexo feminino, idade adulta, sedentários, com excesso de peso e obesidade abdominal. Quanto aos questionários e escala avaliados, alto impacto e 
intensidade de dor importante foram encontrados na maioria dos pacientes, sendo que maiores médias de CC e IMC foram encontradas nos pacientes cujo impacto foi substancial/importante pelo HIT-6, identificando-se associação entre excesso de peso e impacto substancial/importante da dor e tendência de associação em relação à CC. $\mathrm{O}$ excesso de peso ainda foi identificado como fator de risco, aumentando as chances de maior impacto da dor. Por fim, observou-se que a chance de maior impacto da dor também foi maior no sexo feminino e nos indivíduos sedentários desse estudo. Destaca-se a importância de estudos que envolvam os pacientes com enxaqueca, já que esta gera significativas limitações na qualidade de vida.

\section{AGRADECIMENTOS}

À Coordenação e equipe do "Projeto Interdisciplinar de Atenção à Saúde ao Portador de Enxaqueca", pelo apoio e incentivo.

\section{REFERÊNCIAS}

1. Schwedt TJ. Chronic migraine. BMJ. 2014;348:g1416.

2. International Headache Society (IHS). Headache Classification Committee of the International Headache Society (IHS) The international classification of headache disorders. $3^{\text {rd }}$ ed. Cephalalgia. 2018;38(1): 1-211.

3. Queiroz LP, Silva Junior AA. The prevalence and impact of headache in Brazil. Headache. 2015;55(Suppl 1):32-8.

4. Stovner LJ, Nichols E, Steiner TJ, Abd-Allah F, Abdelalim A, Al-Raddadi RM, et al. Global, regional, and national burden of migraine and tension-type headache, 1990-2016: a systematic analysis for the Global Burden of Disease Study 2016. Lancet Neurol. 2018;17(11):954-76.

5. Vladetić M, Jančuljak D, Butković Soldo S, Kralik K, Buljan $\mathrm{K}$. Health-related quality of life and ways of coping with stress in patients with migraine. Neurol Sci. 2017;38(2):295-301.

6. Charles A. The pathophysiology of migraine: implications for clinical management. Lancet Neurol. 2018;17(2):174-82.

7. Amin FM, Aristeidou S, Baraldi C, Czapinska-Ciepiela EK, Ariadni DD, Di Lenola D. The association between migraine and physical exercise. J Headache Pain. 2018;19(1):83.

8. Ornello R, Ripa P, Pistoia F, Degan D, Tiseo C, Carolei A, et al. Migraine and body mass index categories: a systematic review and meta-analysis of observational studies. J Headache Pain. 2015; $16: 27$.

9. Peterlin BL, Rosso AL, Rapoport AM, Scher AI. Obesity and migraine: the effect of age, gender and adipose tissue distribution. Headache. 2010;50(1):52-62.

10. Santos IS, Goulart AC, Passos VM, Molina MC, Lotufo PA, Bensenor IM. Obesity, abdominal obesity and migraine: a crosssectional analysis of ELSA-Brasil baseline data. Cephalalgia. 2015;35(5):426-36.
11. MinistériodaSaúde.Antropometria: comopesaremedir.Brasília: Ministério da Saúde; 2004.

12. World Health Organization. The World Health Report 1998: life in the $21^{\text {st }}$ century. Geneva: WHO; 1998. 241p.

13. Nutrition Screening Initiative, NSI-2002. A physician's guide to nutrition in chronic disease management for older adults. Leawood: American Academy of Family Physicians; 2002.

14. World Health Organization. Obesity: preventing and managing the global epidemic: report of a WHO consultation. WHO technical report series. Geneva: World Health Organization; 2000. $253 \mathrm{p}$.

15. Jensen MP, Karoly P, Braver S. The measurement of clinical pain intensity: a comparison of six methods. Pain. 1986;27(1):117-26.

16. StewartWF, Lipton RB, KolodnerKB, Sawyer J, Lee C, Liberman JN. Validity of the Migraine Disability Assessment (MIDAS) score in comparison to a diary-based measure in a population sample of migraine sufferers. Pain. 2000;88(1):41-52.

17. Kosinski M, Bayliss MS, Bjorner JB, Ware JE Jr, Garber WH, Batenhorst A, et al. A six-item short-form survey for measuring headache impact: the HIT-6. Qual Life Res. 2003;12(8):963-74.

18. Wong RK, Palsson OS, Turner MJ, Levy RL, Feld AD, von Korff $\mathrm{M}$, et al. Inability of the Rome III criteria to distinguish functional constipation from constipation-subtype irritable bowel syndrome. Am J Gastroenterol. 2010;105(10):2228-34.

19. Merikangas KR. Contributions of epidemiology to our understanding of migraine. Headache. 2013;53(2):230-46.

20. Martin VT, Lipton RB. Epidemiology and biology of menstrual migraine. Headache. 2008;48(Suppl 3):S124-30.

21. Straube A, Müller H, Stiegelbauer V, Frauwallner A. Migräneprophylaxe mit einem Probiotikum. MMW - Fortschritte der Medizin. 2018;160(5):16-21.

22. Overath CH, Darabaneanu S, Evers MC, Gerber WD, Graf M, Keller A, et al. Does an aerobic endurance programme have an influence on information processing in migraineurs? J Headache Pain. 2014;15(1):11.

23. Le H, Tfelt-Hansen P, Skytthe A, Kyvik KO, Olesen J. Association between migraine, lifestyle and socioeconomic factors: a population-based cross-sectional study. J Headache Pain. 2011;12(2):157-72.

24. Lima LV, Abner TSS, Sluka KA. Does exercise increase or decrease pain? Central mechanisms underlying these two phenomena. J Physiol. 2017;595(13):4141-50.

25. Blau JN. Migraine: theories of pathogenesis. Lancet. 1992;339(8803):1202-7.

26. Spahis S, Delvin E, Borys J-M, Levy E. Oxidative stress as a critical factor in nonalcoholic fatty liver disease pathogenesis. Antioxid Redox Signal. 2017;26(10):519-41.

27. Goadsby PJ. Pathophysiology of migraine. Neurol Clin. 2019;27(2):335-60.

28. Sadeghi O, Askari G, Maghsoudi Z, Ghiasvand R, Khorvash F. The association between abdominal obesity and characteristics of migraine attacks in Iranian adults. Iran J Nurs Midwifery Res. 2016;21(3):271-7.

29. Chai NC, Bond DS, Moghekar A, Scher AI, Peterlin BL. Obesity and headache: part II - potential mechanism and treatment considerations. Headache. 2014;54(3):459-71.

30. Verrotti A, Di Fonzo A, Penta L, Agostinelli S, Parisi P. Obesity and headache/migraine: the importance of weight reduction through lifestyle modifications. Biomed Res Int. 2014;2014:420858.

Local de realização do estudo: Clínica Escola de Farmácia, Departamento de Ciências da Vida, Universidade do Estado da Bahia (UNEB), Salvador, Bahia, Brasil.

Conflito de interesse: As autoras declaram não haver.

Foi apresentado como trabalho de destaque no XXIII Congresso Brasileiro de Nutrição Parenteral e Enteral, entre 20 a 23 de outubro de 2019, em Foz do Iguaçu-PR. 